\title{
RFID Tag as a Sensor - A Review on the Innovative Designs and Applications
}

\author{
Zhaozong Meng ${ }^{1}$, Zhen $\mathrm{Li}^{2}$ \\ ${ }^{1}$ School of Electrical and Electronic Engineering, University of Manchester, Oxford Road, Manchester M13 9PL, UK \\ zhaozong.meng@manchester.ac.uk \\ ${ }^{2}$ School of Materials, University of Manchester, Oxford Road, Manchester M13 9PL, UK
}

\begin{abstract}
The Radio Frequency Identification (RFID) technology has gained interests in both academia and industry since its invention. In addition to the applications in access control and supply chain, RFID is also a cost-efficient solution for Non-Destructive Testing (NDT) and pervasive monitoring. The battery free RFID tags are used as independent electromagnetic sensors or energy harvesting and data transmission interface of sensor modules for different measurement purposes. This review paper aims to provide a comprehensive overview of the innovative designs and applications of RFID sensor technology with new insights, identify the technical challenges, and outline the future perspectives. With a brief introduction to the fundamentals of RFID measurement, the enabling technologies and recent technical progress are illustrated, followed by an extensive discussion of the novel designs and applications. Then, based on an in-depth analysis, the potential constraints are identified and the envisaged future directions are suggested, including printable/wearable RFID, System-on-Chip (SoC), ultra-low power, etc. The comprehensive discussion of RFID sensor technology will be inspirational and useful for academic and industrial communities in investigating, developing, and applying RFID for various measurement applications.
\end{abstract}

Keywords: RFID sensor, NDT, pervasive monitoring, energy harvesting, SoC.

\section{INTRODUCTION}

As a contactless and non-line-of-sight identification and data transmission technology, RFID has been widely applied for access control and information tracking in logistics and industrial processes [1]. It is also considered as an eminent enabling technology for the realisation of ubiquitous monitoring in Internet of Things (IoT) [2], [3].

Since the inductive coupling or backscattered radio waves in RF identification can be used to detect the physical parameters of tagged objects, RFID technology is also a potential solution for smart sensing to deal with some sophisticated problems [4][5]. For instance, it can be used to discriminate the variation of materials as a transmission medium of the radio waves. Therefore, RFID tags are also innovatively used as electromagnetic sensors for different measurement purposes, such as strain detection [6], [7], material corrosion analysis [8], [9], crack detection [10], [11], [12] and food quality evaluation [13], [14].

In addition, RFID tags can also be integrated with electronic components, such as sensory material, Analogueto-Digital Converter (ADC), and Micro-Controller Unit (MCU) to make an integrated sensor module. The RFID tag is used as a communication interface for data transmission. Passive RFID sensors harvest the RF energy from RF radiation to power the circuit and complete the sensing function, then save the data in the RFID chip to be accessed by RFID readers, which is described in [15], [16], and [17]. The passive sensing is of interest for data collection in remote sensing and RFID Wireless Sensor Network (WSN), such as health monitoring [18] and indoor localisation [19].

The promising prospect of this inexpensive technology has fostered studies in many disciplines. The rapid progress of RFID sensors and the lack of systematic description of this technology in the literature are the causes of concerns, which have motivated this investigation to bridge the gap by providing a comprehensive overview of the novel designs and applications with new insights.

The structure of this paper is organised as follows: Section 2 introduces the fundamentals of RFID measurement, Sections 3 and 4 illustrates the enabling techniques and the state-of-the-art designs and applications, Section 5 summarises the underlying challenges and suggests the future directions, and finally Section 6 concludes the work.

\section{FUNDAMENTALS OF RFID MEASUREMENT}

Normally, the RF identification procedure between an Ultra-High Frequency (UHF) RFID reader and a tag as a transponder is as depicted in Fig. 1 [20], where CW and RCS mean continuous wave and radar cross-section respectively. 
Since RFID sensing is an extended function of RF identification, it fulfills the measurement function by taking advantage of variation in inductive coupling and RF backscattering caused by the objects under measurement. This section illustrates the methods of utilising RFID tags as sensors and their fundamentals.

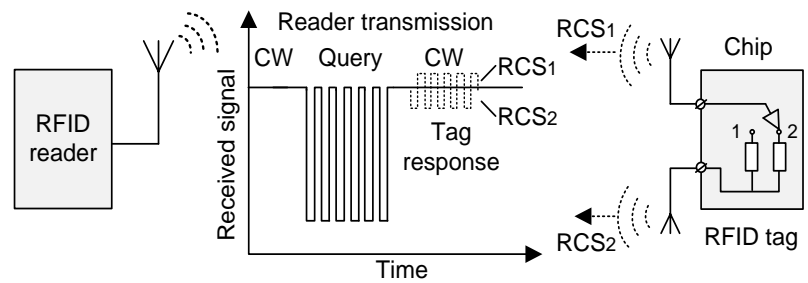

Fig.1. Diagram of UHF RFID reading fundamentals.

\section{A. Basics of RFID technology}

The RFID operates at a variety of frequencies which are summarised in Table 1. Both Low Frequency (LF) and High Frequency (HF) RFID operating in the near field and energy transfer is through inductive coupling. However, for UHF and higher like Super High Frequency (SHF) within microwave frequency range, the communication and energy transfer is in the far field through backscattering.

Table 1. Frequencies and reading range of RFID techniques.

\begin{tabular}{|c|c|c|}
\hline $\begin{array}{c}\text { RFID } \\
\text { Techniques }\end{array}$ & $\begin{array}{c}\text { Operating } \\
\text { Frequencies }\end{array}$ & $\begin{array}{c}\text { Free Space } \\
\text { Reading Range }\end{array}$ \\
\hline LF & $125-134.3 \mathrm{kHz}$ & $<10.0 \mathrm{~cm}$ \\
\hline HF & $13.56 \mathrm{MHz}$ & $<1.0 \mathrm{~m}$ \\
\hline $\mathrm{UHF}$ & $860-960 \mathrm{MHz}$ & $1.0-12.0 \mathrm{~m}$ \\
\hline SHF & $2.45-5.8 \mathrm{GHz}$ & Up to $100.0 \mathrm{~m}$ (Active) \\
\hline
\end{tabular}

For measurement, LF and $\mathrm{HF}$ are used more in metal materials and UHF and higher are used more for dielectric materials weaker in conductivity, such as concretes and food. Some RFID sensors are custom-designed, while some are compliant with certain global regulatory such as Electronic Product Code (EPC) Class 1 Generation 2 for UHF RFID [21]. The use of a fully standardised technology UHF RFID EPC Class 1 Generation 2 can benefit from plenty of Commercial Off-The-Shelf (COTS) components.

\section{B. RFID tag as an electromagnetic sensor}

The fundamentals of LF/HF and UHF RFID tags measurement are as shown in Fig. 2, and Fig. 3 represents the equivalent circuit of LF/HF RFID near-field coupling.

Basically, an RFID tag consists of an L-C-R parallel circuits. When an RFID antenna is put on a measurement object as a transmission medium of radio waves, its electrical properties $L_{2}$ and $C_{2}$ are changed. Assume the transformed resistance of the tag is $Z^{\prime}$, the resonance frequency of the tag at the maximum point of the real part of $Z$ ' can be represented by:

$$
f_{0}=\frac{1}{2 \pi \sqrt{C_{2} \cdot L_{2}}}
$$

$\mathrm{R}_{2}$ in the circuits decides the bandwidth of resonance. When there is variation in $C_{2}$ and $L_{2}$ caused by the measured object, $f_{0}$ shifts.

In addition, Q-factor of the tag which is assumed to be a simple parallel resonant tank is defined by:

$$
Q=R_{2} \sqrt{\frac{C_{2}}{L_{2}}}
$$

Therefore, by determining the $f_{0}$ and $Q$, and analysis of the variation in $L_{2}, C_{2}$, and $R_{2}$, the electrical properties of measured objects can be obtained. This basic theory is explored in different ways for measurement purposes. For example, a material sensitive to moisture can be located on the substrate of a RFID tag to affect the electrical properties $C_{2}$ and $L_{2}$. The moisture content can be obtained with a calibration procedure. UHF measurement based on RF backscattering RFID are similar in fundamentals.

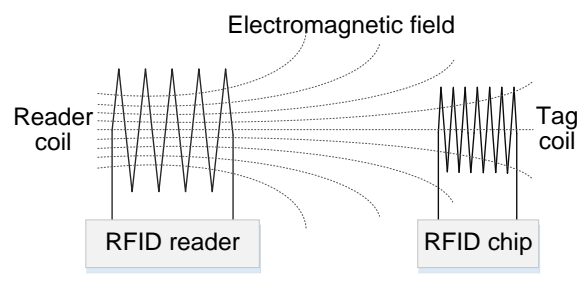

a) LF/HF RFID

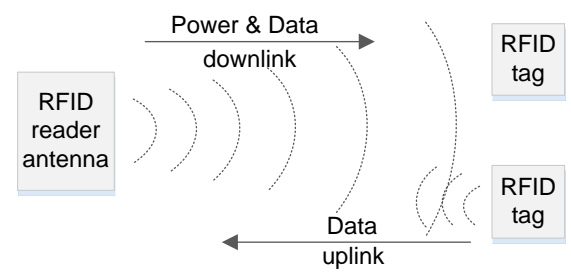

b) UHF RFID

Fig.2. LF/HF and UHF RFID tag sensing.

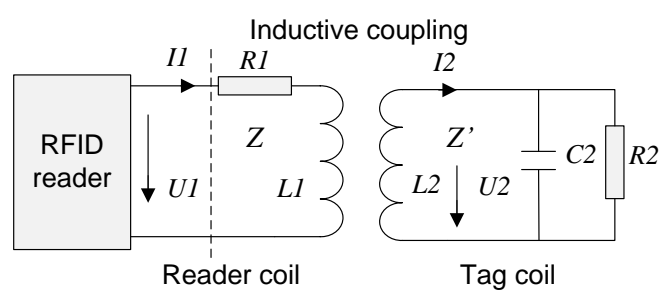

Fig.3. Equivalent circuit of LF/HF RFID near-field coupling.

\section{RFID tag as an energy harvesting and data transmission medium}

In addition to taking advantage of resonance frequency of the RFID antenna for measurement, the capability of RF energy harvesting for data transmission is another promising technique, which allows the integrated sensor module to be able to perform passive, batteryless and remote sensing.

To achieve the functions, an RFID antenna with matching network is connected to a RF-to-DC rectifier, which 
generates DC voltage from RF signal. A charge pump with a voltage supervisor controls the charging and discharging of a storage capacitor. When the storage capacitor is charged, the voltage supervisor starts to discharge and power the MCU which collects sensor data and writes to particular data banks of the RFID chip that are readable by RFID readers. A reference circuits design is given in Fig. 4.

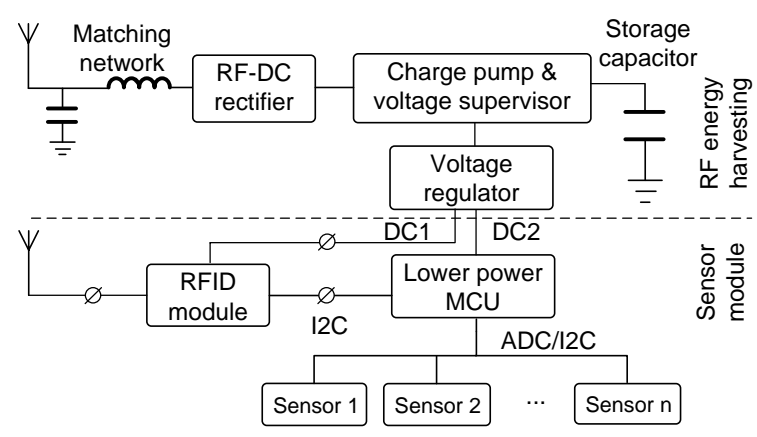

Fig.4. A reference design of UHF RFID sensor.

The passive RFID tag is a promising candidate to integrate with an ultra-low power sensor to constitute a batteryless RFID sensor. Some commercial RF-to-DC converters such as Powercast P2110 [22], I2C UHF RFID Gen2 ICs such as Impinj Monza X-2K, ultra-low power MCUs such as TI MSP430 serials are key enabling technologies [15]. The integration of sensor module and RFID module has allowed the sensors to transmit data conveniently in a wireless way, and is therefore widely investigated and applied [23].

Generally, RFID sensors are based on the two fundamental methods described above and their further extensions, and numerous novel designs and applications are conducted accordingly.

\section{RFID sensing technology classification}

RFID sensors achieve measurement tasks in different ways. According to operating fundamentals, the related methods of RFID measurement can be classified into four categories: RFID electromagnetic sensor, RFID tag integrated sensor, RFID tag array, and RFID sensor networks. The former two kinds are commonly used, and the latter two are the functional extensions of the former. The different kinds and their fundamentals of measurement are given in Table 2 .

Table 2. Categories of RFID sensor measurement.

\begin{tabular}{|l|l|}
\hline \multicolumn{1}{|c|}{ Categories } & \multicolumn{1}{c|}{ Fundamental of Measurement } \\
\hline $\begin{array}{l}\text { RFID } \\
\text { electromagnetic } \\
\text { sensor }\end{array}$ & $\begin{array}{l}\text { Normal passive RFID tag or chipless RFID, for } \\
\text { which measurement is based on the analysis of } \\
\text { its spectral or phase characteristics }\end{array}$ \\
\hline $\begin{array}{l}\text { RFID tag } \\
\text { integrated sensor }\end{array}$ & $\begin{array}{l}\text { Integrated with a sensor module, RFID is used } \\
\text { for energy harvesting and data transmission }\end{array}$ \\
\hline RFID tag array & $\begin{array}{l}\text { RFID array for expanding measurement space or } \\
\text { for localisation and tracking }\end{array}$ \\
\hline $\begin{array}{l}\text { RFID sensor } \\
\text { networks }\end{array}$ & $\begin{array}{l}\text { Batteryless and low-cost solution for wireless } \\
\text { sensor networks monitoring }\end{array}$ \\
\hline
\end{tabular}

A plethora of studies on measurement of strain, crack, corrosion, temperature, moisture, gas, blood glucose, etc., localisation, and RFID WSN monitoring fall in the four categories. The prosperity of different kinds of RFID sensing technology is also the result of continuous progress of enabling techniques to be discussed in Section 3.

\section{ENABLING TECHNIQUES OF RFID SENSORS}

According to the fundamentals of RFID measurement described in Section 2, the advantages of RFID sensing are introduced by techniques of multi-disciplines, such as RF and antenna, RF identification components, energy harvesting, and integration of ADC and low power digital components. As one of the research focuses which are promising in practical applications, RFID technology has attracted many research efforts. This section illustrates the major enabling technologies that offer the RFID sensors with the addressed advantages.

\section{A. Innovation in RFID antenna design}

Antenna is a critical component of RFID tags. Its performance can be improved by introduction of new structures and materials. For example, in terms of shape of antenna, a novel 3-D cubic antenna for wireless sensor networks and RFIDs for environmental sensing is reported in [24], and this cubic design has achieved nearly isotropic pattern. In [25], an ultra-low-cost RFID tag is presented, which comprises a soft magnetic ribbon that is biased by an adjacent permanent magnet. The tag is read by measuring changes in the ribbon's magnetisation while applying magnetic AC and DC fields. With respect to material of antennas, a printable RFID antenna with low temperature processing of graphene ink is introduced in [26], and its feasibility has been demonstrated for low-cost printable RF applications. A highly conductive textile-based elastic RFID tag antenna is fabricated in [27] to introduce elasticity, flexibility, and mechanical strength. In addition, a flexible magnetic composite material is introduced for wearable RFID antenna [28], which potentially has enabled the significant miniaturisation of RFID antennas in UHF frequency band.

These novel designs of RFID antenna have either improved the performance of RFID tags or extended their scope of applications.

\section{B. Chipless RFID}

Rather than to embed silicon integrated circuits for ID extraction, chipless RFID uses an electromagnetic signature for data encoding to reduce the cost of RFID tags [29]. The challenges facing the design of a chipless RFID transponder is how to perform data encoding without the presence of a chip. To this end, two general types of RFID transponders can be identified: Time-Domain Reflectometry (TDR)-based and spectral signature-based. The former transponder is interrogated by sending a signal with the reader in the form of a pulse and listening to the echo of the pulse sent by the tag, while the latter decodes spectrum features of RFID tags encoded using resonant structures. A Ultra-Wideband (UWB) impulse radar based reader is presented in [30], 
which interrogates a chipless tag with a UWB pulse and analyses received backscatter in the time domain to obtain the IDs. A chipless RFID transponder based on phase encoded backscatter is proposed in [31], which comprises 3 microstrip patch antennas loaded with open circuited high impedance stubs. The antennas re-radiate backscattered signals with distinct phase characteristics that are encoded as hexadecimal bits for the proposed chipless RFID tag. A typical study on spectral signature-based RFID is to apply exciting RF to recognise metallic strip letters on dielectric substrate [32]. Results demonstrate the possibility to uniquely identify the alphabet with high certainty by observing the resonance peaks.

From the fundamentals and example applications described above, both TDR-based and spectral signaturebased chipless RFIDs are good candidates for RFID electromagnetic sensors.

\section{RF energy harvesting and hybrid power supply}

$\mathrm{RF}$ energy harvester or RF-to-DC converter is a critical component in RFID tags, the function of which is to convert RF signal into DC voltage that can be used to power low voltage electronic devices. In order to fix the efficiency drop problem of Dickson's circuits due to turn-on voltage of diodes, the Metal-Oxide-Semiconductor Field-Effect Transistor (MOSFET) diode based Complementary MetalOxide-Semiconductor (CMOS) RF-to-DC converter is investigated in order to generate stable DC power with an appropriate voltage level [33]. In addition, some studies introduce novel hybrid powering solutions, such as semipassive solar-powered temperature sensor based on a timecoded UWB RFID tag, which is built up with simple COTS components [34]. In order to extend the reading range, optional battery is also included for some designs.

Both energy harvesting and hybrid power supply methods are effective approaches to extend the batteryless sensing advantage of RFID based sensing devices.

\section{SoC RFID sensor}

In addition to energy efficiency, miniaturisation is also a key parameter of RFID based applications. As shown in Fig 5 , integration of RFID technique with other modules on an integrated chip as a SoC device is a prospective direction and heuristic studies have already started. Investigation of integrating sensing materials on $0.18 \mu \mathrm{m}$ CMOS becomes common in laboratory studies [36], [37]. Highly integrated ICs with RF harvesting, DC regulator, ADC, and MCU are the future of RFID sensors and other wireless sensor nodes.

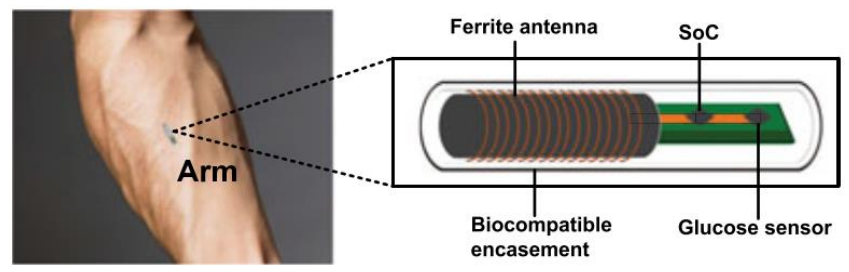

Fig.5. Implantable SoC RFID for glucose monitoring, [35].

\section{E. Printable/wearable RFID}

Printable and wearable RFID that is compliant with available technologies and the safety standards is highly demanded. Printable RFID is a solution to produce low-cost and environment friendly RFID tags for identification and sensing applications. One promising solution is to use inkjet-printed RFID circuits on flexible and paper substrates to provide RFID sensing functionalities, which is as shown in Fig. 6 [2]. This method is more economical and environment friendly compared to traditional methods. Development of wearable RFID tags involving biomonitoring of human still remains challenging as the interaction of antenna with the human body results in efficiency and sensitivity degradation of RFID sensing. The effects of human body on a wearable UHF RFID is examined in [38] focusing on variation of antenna-body separation distance on the antenna properties. The measured three-dimensional radiation pattern is useful to peer investigations. A tag geometry combining folded conductors and tuning slots with numerical analysis and experiments is presented in [39], which has achieved a design applicable to any part of human body. Evidently, the printable and wearable RFID techniques have also expanded the application scope of conventional RFID tags.

The techniques either for improving the performance of RFID tag, or for integration of RFID with other disciplines have finally contributed to the RFID sensor technology. The continuous progress of enabling techniques in the above aspects has extended the functions of different RFID sensing technologies for measurement purpose and fostered some new applications in a variety of fields.

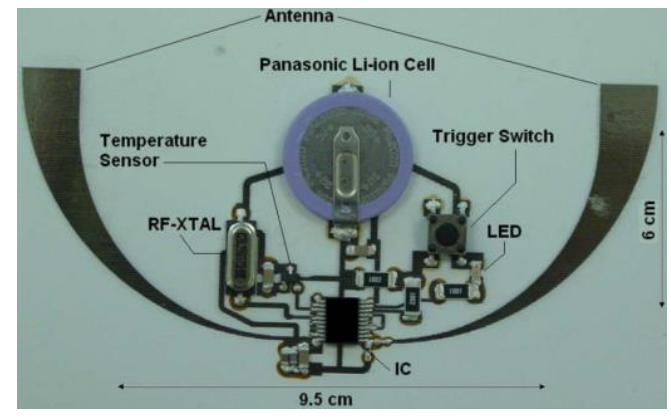

Fig.6. Inkjet-printed RFID sensor on paper substrate, [2].

The techniques either for improving the performance of RFID tag, or for integration of RFID with other disciplines have finally contributed to the RFID sensor technology. The continuous progress of enabling techniques in the above aspects has extended the functions of different RFID sensing technologies for measurement purpose and fostered some new applications in a variety of fields.

\section{INNOVATIVE DESIGNS AND APPLICATIONS OF RFID SENSORS - A REVIEW OF THE STATE OF THE ART}

With the continuous progress of enabling techniques, many novel designs and innovative applications of RFID sensor technology are reported in the literature. This section gives a brief introduction to the application areas, and 
classifies the reported designs and applications with the illustrations of some typical examples.

Batteryless, miniaturised, remote sensing, low-cost, easy fabrication and integration are key advantages identified that make RFID sensors accepted in a wide range of applications. From the perspective of application fields, the categorisation of RFID sensors and the variables to be measured in order to fulfil the functions are given in Fig. 7.

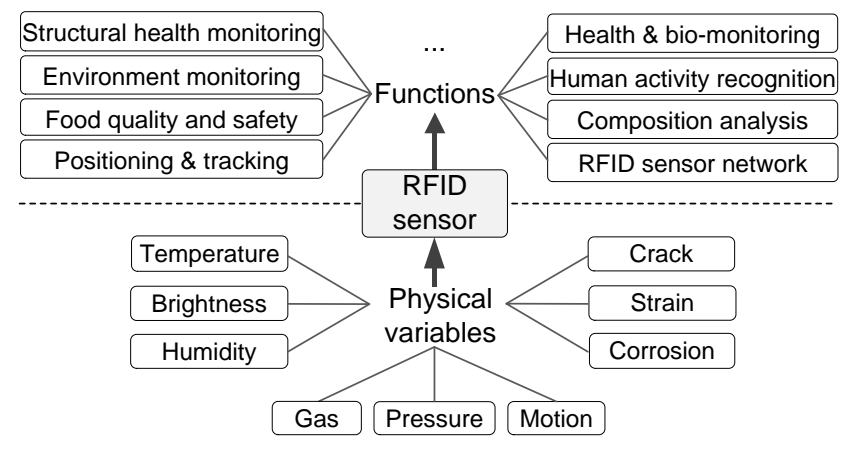

Fig.7. Applications of RFID sensors.

\section{A. Structural health monitoring (SHM)}

Monitoring mechanical conditions of critical structures so as to prevent catastrophic failure is a very significant task. RFID sensors that can be used for passive, non-destructive and remote evaluation are widely applied. Commonly, the structure under test is examined by measurement of deformation, crack, corrosion, and strain. For high conductivity materials like metals and carbon fibre composites, the penetration of electromagnetic signals is limited. Thus, only the surface damage can be detected [12].

With respect to crack detection, a Surface Crack Antenna Reflectometric Sensing (SCARS) is introduced in [10], which implements chipless RFID for pervasive and wireless detection to identify the length and orientation of surface crack in structural materials. A crack width RFID sensor based on high sensitivity phase detection of passive RFID is presented in [11]. The described sensor can detect submillimetre deformation occurring on the object. In addition, an RFID antenna for detection of surface crack in civil infrastructure is introduced in [40], which also proposes a solution to improve spatial coverage with 2-D grid of tags.

In terms of corrosion detection, LF RFID is applied to characterise steel corrosion in [8], and experiments demonstrate the sensitivity of this RFID Wireless Power Transfer (WPT) solution. An RFID based sensor for corrosion monitoring of reinforced concrete structures is presented in [9], which can perform linear polarisation, open circuit potential and temperature measurements. Test results can show the initiation and progression of corrosion with obtained linear polarisation resistance measurement. In addition, a batteryless RFID based embedded sensor is proposed for long-term structural health monitoring in [41] to monitor the corrosion, temperature, and humidity level in reinforced concrete structure.

RFID sensor strain detection is also widely investigated. Intel Wireless Identification and Sensing Platform(WISP) is modified to interface with a foil resistance strain gauge for uniaxial tension tests of carbon fibre composites [6]. The results show excellent agreement with the prediction of NASTRAN finite element model. A Breakage-Triggered (BT) strain sensor integrated with an RFID tag for wireless communication is designed to perform non-contact scanning of structural deformation condition [7]. This system can rapidly identify the spot where the stain has surpassed the threshold pre-set by engineers and decision makers. A flexible and stretchable inductor-capacitor (LC) resonator based chipless RFID tag fabricated by stamping with silver nano ink for strain sensing is presented in [42]. A single tag and multiple tags identifications are achieved by changes in resonance frequency, and results agree well with theoretical calculation. A dual-interrogation-mode method combining chipped and chipless RFID to detect embroidered RFID strain sensor is described in [43]. This work validates a dual interrogation mode and proves that chipped RFID sensor tags can be detected accurately utilising backscattering RCS measurements.

According to the above studies, passive RFID is widely applied for non-destructive testing, and the principle of measurement is to identify the shift of resonance frequency due to variation of structural conditions. There is also investigation that uses $2 \mathrm{D}$ array to extend spatial coverage. The application of battery free RFID sensor has alleviated the constraints caused by various measurement conditions, which has been provided a promising and inexpensive remote sensing solution for structural health monitoring.

\section{B. Environment monitoring}

RFID sensors are also applied for environment monitoring mainly on measurement of gas, humidity, and temperature.

Gas detection is a typical case of environment monitoring applications. A batteryless UHF RFID tags coated by different sensitive layers for volatile compounds detection and ambient sensing is described in [44]. Results are encouraging while packing four RFID sensors into a compact array still remains an open issue due to an increase in cross sensitivity of each tag. A single-wall Carbon Nanotube (CNT) buckypaper based RFID tag as a low-cost and maintenance free sensor solution for $\mathrm{NH}_{3}$ measurement is investigated and verified by experiments in [45]. An inkjet printing method to deposit single-walled CNT film on a fully printed UHF RFID module on paper to form a wireless gas sensor node for toxic gas detection is introduced in [46]. This work demonstrates the feasibility of inkjet-printed CNT for RFID enabled sensor nodes.

For humidity measurement, [47] presents a prototype passive wireless UHF RFID sensor for humidity monitoring in a built environment through far-field backscatter coupling. This sensor is implemented by incorporating a humidity sensitive polyimide film onto top surface of RFID tags. Some proactive investigations attempt to integrate sensor elements to RFID chips to build SoC modules. A UHF RFID tag with ultra-low power, small size, high resolution temperature sensor adopting a double Voltage Controlled Oscillator (VCO) is fabricated using the SMIC CMOS 0.18 $\mu \mathrm{m}$ EEPROM 2P4M process [48]. These properties allow the use of the RFID tag as a batteryless sensor for a long-range wireless temperature monitoring. In [49], a capacitance humidity sensor with a co-integrated 
energy efficient Capacitance-to-Digital Converter (CDC) which is implemented on a $0.16 \mu \mathrm{m}$ CMOS technology is presented. Measurement results prove that it achieves a resolution of 12.5 bits with a measurement time of $0.8 \mathrm{~ms}$, while drawing only $8.6 \mu \mathrm{A}$ from $1.2 \mathrm{~V}$ power supply which outperforms the state-of-the-art in capacitive-sensor interfaces in terms of energy efficiency. An RFID based capacitive humidity sensor tag fabricated by $0.18 \mu \mathrm{m}$ CMOS process with a top metal layer deposited to form a interdigitated electrodes filled with polyimide as humidity sensing layer is presented in [50]. With $0.5 \mathrm{~V}$ power supply, results show excellent linearity, hysteresis and stability. In addition, printed LC resonator structures are also investigated for humidity detection. A two planar LC resonators RFID humidity sensor tag operating wirelessly through inductive coupling for short-range item tracking and humidity monitoring is presented in [51]. The RFID humidity sensor provides excellent sensitivity and reasonable response time to humidity. Applying the fundamentals of RFID tag resonance frequency shift measurement, an inductor coil and an interdigitated capacitor are developed by screen printing and inkjet printing to form an LC resonator for humidity detection in [52].

With respect to RFID temperature sensor, there are plenty of investigations reported in the literature. An investigation of RFID sensor application for soil solarisation purposes is presented in [53]. RFID temperature sensors are evaluated and results demonstrate that it is an easy-to-use and cheap tool to support the decision-making process during longterm treatment like solarisation. A $0.18 \mu m$ CMOS SoC passive RFID tag with an embedded temperature sensor for UHF EPC Gen2 is introduced in [54]. The proposed gaincompensation technique and low-power time-readout scheme decrease sensing error and power consumption.

From the above studies, it is found that integration of RFID tag with various sensing techniques is effective and promising way for environment monitoring applications.

\section{Food quality and safety}

Food quality and safety is an area electromagnetic sensors are preferred since they can test the internal of food and satisfy the hygienic requirements. The inexpensive RFID sensor solution is a competitive alternative for food quality assessment. Examples of applications include monitoring of freshness of fruit, milk, fish, and bacterial growth, etc. [14].

An approach to modify RFID tag with chemically sensitive conductive composites is introduced in [55] to detect different biogenic amines associated with food spoilage. The RFID response is then dependent on amine concentration, tag initial resistance, and type of biogenic amine. UHF RFID is applied to detect contamination and meat quality in [13]. The contamination of food can be obtained, provided the variation of food permittivity over time due to aging is known. An RFID sensor system for vegetable freshness monitoring is proposed in [56], which is designed by integrating an oxygen and carbon dioxide concentration sensor with a RFID tag for wireless data transmission. In a similar way, an HF RFID based sensor for monitoring of freshness of packaged vegetables based on measurement of temperature and humidity is presented in [57], which has extended the reading distance to $30 \mathrm{~cm}$.

Differently, a multi-sensor RFID tag uses both HF and UHF in [58], which can check quality of food and also monitor the distribution of food with the two frequency bands. An ultra-low power CMOS temperature sensor is proposed targeting at RFID food monitoring applications by employing serially connected sub-threshold MOS as sensing element in [37], which reaches $119 n \mathrm{~W}$ power consumption at room temperature. Validation study on milk freshness is conducted, and model equations are obtained based on experimental studies. In addition to individual measurements, the RFID sensor is also integrated with WSN technology for food quality monitoring and control in a wireless network with databases [59], [60].

Since food materials are usually not bulk materials of regular shapes, RFID is seldom used as an electromagnetic sensor but more for data transmission of integrated sensors for $\mathrm{PH}$, temperate, and moisture content, etc.

\section{Positioning and tracking}

Due to the strengths in non-line-of-sight, object positioning and tracking based on RFID technology is another widely accepted use case, including the localisation and tracking of vehicles, equipment, robots, and human, etc.

An RFID positioning approach for connecting vehicles as an alternative when GPS is not available is proposed in [61]. In this approach, RFID tags are installed on the road surface and a tag reader is on-board in vehicles. Low-cost and reasonable accuracy are the strengths of this solution. A Real-Time Location System (RTLS) for hospital equipment tracking with RFID technique is presented in [62]. The system utilises passive RFID tags mounted on flooring plates and several peripherals for sensor data interpretation and achieves the desired accuracy.

A partially observed feedback controller for a wheeled mobile robot is presented in [63], where the feedback signal is in the form of noisy Received Signal Strength Indicator (RSSI) emitted from RFID tags. The proposed controller requires neither an accurate mapping between the line-ofsight distance and the RSS measurement, nor the linearisation of the robot model. In [64], differential evolution approach is applied in an RFID sensor deployment for mobile robot localisation. An RFID based system performs both real-time monitoring of body temperature and location of the body is demonstrated in [65], where the RFID chip's integrated sensor is used for temperature detection, and reference tags and a multiple-antenna time division multiplexing system are used for localisation with a K-Nearest Neighbours (KNN) algorithm.

For RFID positioning and tracking, there may be uncertainties, gap, and errors due to the speed of objects or the arrangement of RFID tag array. The calibration methods and algorithm to eliminate these errors are critical to the accuracy of measurement. Least Mean Square (LSM), Interactive Multiple Model (IMM)-based global fusion, Kalman Filter algorithm, hierarchical fusion algorithm, Heron-bilateration location estimation, etc. are investigated [19], [66], [67]. In order to solve the multi-path phenomenon of RSSI based RFID indoor localisation, algorithms SA-LANDMARC and COCKTAIL are proposed 
to improve accuracy and scalability for RFID WSN localisation in [68], and the accuracy can reach $0.7 \mathrm{~m}$ and $0.45 m$ respectively.

\section{E. Health monitoring and bio-monitoring}

Newly invented digital health devices have emerged frequently in recent years. Applications of RFID for health monitoring and bio-monitoring have also begun to happen.

One direction is to take advantage of the strength of RFID sensor in remote sensing for health parameter monitoring. A set of frameworks based patient life cycle and time-andmotion perspectives is introduced in [69] for making use of RFID information system to offer benefit for patient care and hospital operations. An investigation combining RFID real-time remote monitoring of body temperature and the location is introduced in [65], which is considered to be of interest in disaster relief. A long range UHF RFID sensor designed using $0.35 \mu \mathrm{m}$ CMOS standard process is presented in [70], which allows an ID and a temperature reading range of $2 m$ from a $2 W$ effective radiated power reader. Test results demonstrate potential of the sensor as a batteryless solution for wireless human body temperature monitoring.

Another direction is to innovatively make use of the electromagnetic field of RFID tag for measurement. A wireless powered implantable electromagnetic sensor tag for continuous blood glucose monitoring is presented in [35], which is remotely powered by $13.5 \mathrm{MHz}$ RFID for sensing and communication. This system can produce reliable and accurate measurement of glucose, which is of interest by clinical and patient care. An epidermal passive strain sensor using UHF RFID tags is presented in [71], which intends to detect eyebrow or neck skin stretch to offer the possibility of allowing paraplegic patients to control wheelchairs. In [72], an adhesive RFID sweat sensor bandage is reported, which can be made completely intimate with human skin for chronological monitoring of biomarkers in sweat.

There are many innovative applications for RFID health monitoring and bio-monitoring, and the studies in this field will go broader and deeper in the future.

\section{F. Human activity recognition}

Another potential application is human motion detection which is of interest for hospital patient care and elderly care. A single passive body-worn RFID sensor attached over clothing for recognising activities, such as walking and transfers out of beds or chairs in the context of ambulatory monitoring is reported [73]. A prototype sensor-enabled RFID system consists of RFID tags paired with proximity and movement sensors for arm activity monitoring is presented in [74]. Test results demonstrate the reliability and validity in individuals with unimpaired movements. A new tag geometry combining folded conductors and tuning slots which also includes a passive motion detector is introduced in [39]. The measured performance indicates a possible application of these body-worn tags for continuous tracking of human movements. A study on wearable RFID based system for real-time activity recognition is conducted in [75], where recognition is realised by exploiting RFID radio patterns towards easy-to-use solution and high detection coverage. In addition to custom-designed systems, there are specific RFID based platforms suitable for human activity detection, such as iGlove, iBracelet, and WISP [76]. Compared to visual based activity recognition, strengths of RFID based system lie in low-cost and privacy protection.

\section{G. Composition sensing and analysis}

Although not widely investigated, it is an innovative solution to apply RFID sensors for material composition analysis. The conventional passive HF RFID tag is applied for chemical sensing in [77] by coating the RFID tag with chemically sensitive films to form a chemical sensor. By using multivariate statistical analysis tools, the sensor is capable for position independent analyte quantification. Chipless UWB RFID is applied for non-destructive wireless concrete quality measurement in [78]. The measurement is based on detection of the delay between two scattering modes induced by permittivity changes in the concrete, and composition of concrete can be remotely detected and classified. Since electromagnetic wave of RFID is sensitive to materials of different permittivity, composition analysis with RFID is feasible. However, a systematic calibration of the measurement might be a challenging procedure.

\section{H. RFID sensor network}

There have been numerous efforts applying RFID to form a sensor network. By integrating the RFID tags as sensor nodes, an RFID sensor network can be built to perform various measurement tasks. The architecture and technical issues of integrating RFID into WSN is analysed in [79], and three forms of new architectures are proposed with discussions of the feasibility and technical challenges. An RFID sensor network is introduced in [68] to solve the environment factors and multi-path problem in RSSI based localisation. The system consists of a few nodes, each of which acts as both transmitter and receiver. The final location of target is estimated by using the RSSI relationship between the target tag and candidate reference tags. The system design of a UWB-RFID network for tag localisation in IoT applications is presented in [80], and it is found that the architectural choice is strictly application dependent, and must account for costs, complexity, energy efficiency, backward compatibility and performance. It is evident that the architecture design is significant to the success of an RFID sensor network application.

From the above discussion, we can find that RFID sensor technology becomes more and more prosperous. It has penetrated into numerous disciplines and integrated with a variety of new emerging research directions and application fields. The innovative applications of RFID sensors have significantly facilitated and sometimes revolutionised the measurement and monitoring solutions.

\section{DISCUSSION}

From the applications illustrated in Section 4, RFID tag is already considered as a very useful electromagnetic sensing solution. It can be easily integrated with sensing materials and electronic systems for applications using conventional RFID readers and antennas. Using RFID tag as either an electromagnetic sensor or a medium for power and sensor data transmission, they both can take advantage of the RFID 
tags in low-cost, batteryless, miniaturised remote sensing, and easy fabrication.

The application of RFID sensors is not limited to obtaining measurement results through electromagnetic resonance or integrating with sensing components for wireless data transmission. Investigations are bidirectional: (1) from micro-view, innovative designs have invaded to CMOS integrated circuits design to integrate sensitive material and RFID chips; (2) From macro-view, RFID sensors are used to form $2 \mathrm{D}$ arrays for different measurement purposes. It is also attempted to integrate with WSN towards pervasive systems for long-term and large-space monitoring.

\section{A. Technical challenges}

Together with the progress of RFID sensing methods and like any newly developed technologies, some intrinsic challenges that hinder its further developments and applications exist, which are summarised as follows:

1. Calibration for accurate measurement

As is the case with any electromagnetic sensors, the RFID sensor is sensitive to ambient environment and object under test, such as temperature, ambient metal and electromagnetic radiation, and size and shape of object under test, which interact with reflection, refraction and scattering of radio waves. Suffering from the environmental affects is common to resonance based electromagnetic sensors. Therefore, the calibration is an essential but sophisticated step to achieve desired accuracy in measurement.

2. Sparsity and noise of RFID sensing

RFID sensors can perform real-time measurement for static and low speed variations. Data stream from RFID sensors is characterised by sparsity and noise due to the inherent attributes of RFID reading, which makes streaming based high-speed continuous measurement a challenging task.

3. Electromagnetic coupling in RFID sensor array

RFID array is a way to extend the spatial dimension of RFID sensor measurement, and it is a low-cost solution for 2D electromagnetic measurement. However, the electromagnetic coupling between tags results in cross sensitivity, which harms the accuracy of measurement.

4. Security for some use cases

For many sensor occasions such as healthcare assisted diagnosis, and smart home, the parameters measured might be sensitive and confidential for users. Thus, the data protection for these use scenarios is a critical issue for the systems. Since RFID sensors work in distance, security of data is an issue to be considered for some applications.

Corresponding solutions to overcome the technical challenges are being investigated and the open issues are major concerns of future studies in the related areas.

\section{B. Future perspectives}

RFID sensing will stay as a hot research topic in the envisaged future. It will be integrated with new emerging technologies in material science, integrated circuits, and computer science and applied in some new fields. Some commercial components such as integrated RF-to-DC converter, programmable RFID chips, integrated SoC RFID reader chip become common, which will also promote the innovative designs and applications. The major studies of RFID sensor in the future might fall in the following topics:

1. Performance improvement of conventional RFID

Investigations of new RFID tags to improve the performance of RFID measurement for some special use scenarios or for common uses is a promising direction, such as low-cost tags, long distance reading solutions, data security and trust for sensitive applications, etc. For measurement purpose, integration of RFID chips with sensitive materials is a promising solution for passive and wireless measurement.

\section{SoC and energy efficient RFID sensors}

Integration and miniaturisation are essential parameters to electronic systems including sensors, and SoC is a very promising solution. The integrated SoC RFID circuits with ADC and sensitive materials are of interest for many applications. This is also an effective way to reduce energy consumption of the system. The miniature, ultra-low power, passive, and non-contact sensor solution can be extended to many different fields of measurement.

\section{Wearable and bio-monitoring RFID sensor}

Some flexible and foldable materials for fabrication of RFID antenna and substrates are enabling techniques for the wearable RFID tags. Since RFID is light-weight and small, it becomes a promising solution to stick on or embed in human body for the measurement of biomedical parameters for health monitoring and elderly care.

\section{WSN, body area network, and IoT applications}

RFID is considered a key building block for future IoT world, where sensors are connected for pervasive monitoring and control. RFID sensor based WSN is a promising technical approach for collaborative optimisation in some light weight monitoring systems. Body area network is a typical use case. The versatile, low-cost, and miniature RFID sensors will be of interest and play an important role in the IoT ubiquitous sensing environments.

Investigations of new materials for RFID tag, SoC systems and their applications, and the integration with new emerging technologies and computing paradigms are research focuses and key promising directions of RFID sensor technologies in the future.

\section{CONCLUSION}

The rapid progress of batteryless RFID sensors has created tremendous opportunities for wireless measurement in a variety of areas. Applications that utilise an RFID tag as a sensor become common and some novel applications for different use cases appear overwhelmingly. Focusing on this prevalent research direction, this review paper illustrates the fundamental of RFID sensors and the enabling techniques, classifies the novel applications with typical examples, discusses the technical challenges, and outlines the future perspectives of this particular research area. This review is a timely supplement to the literature regarding RFID sensors.

Based on the reported designs and applications, we can envisage that the integration of RFID technology with many fields of science and engineering, and their practical applications will continue to expand deeply and broadly. Especially due to the strengths of low-cost, easy-to-use, and convenient integration, RFID sensors will play a very 
important role in pervasive IoT applications in the future. The comprehensive overview of the innovative studies and in-depth analysis reported in this work will be of interest for academic and industrial communities in investigating, developing, and applying RFID for measurement purposes.

\section{ACKNOWLEDGMENT}

The authors would like thank all staff and students in the microwave sensor laboratory of University of Manchester.

\section{REFERENCES}

[1] Weinstein, R. (2005). RFID: A technical overview and its application to the enterprise. IT Professional, 7 (3), 27-33.

[2] Lakafosis, V., Rida, A., Vyas, R., Yang, L., Nikolaou, S., Tentzeris, M.M. (2010). Progress towards the first wireless sensor networks consisting of inkjet-printed, paper-based RFID-enabled sensor tags. Proceedings of the IEEE, 98 (9), 1601-1609.

[3] Meng, Z., Lu, J. (2016). A rule-based service customization strategy for smart home context-aware automation. IEEE Transactions on Mobile Computing, 15 (3), 558-571.

[4] Occhiuzzi, C., Paggi, C., Marrocco, G. (2011). Passive RFID strain-sensor based on meander-line antennas. IEEE Transactions on Antennas and Propagation, 59 (12), 4836-4840.

[5] Dey, S., Saha, J.K., Karmakar, N.C. (2015). Smart sensing: Chipless RFID solutions for the Internet of Everything. IEEE Microwave Magazine, 16 (10), 2639.

[6] Gasco, F., Feraboli, P., Braun, J., Smith, J., Stickler, P., DeOto, L. (2011). Wireless strain measurement for structural testing and health monitoring of carbon fiber composites. Composites Part A: Applied Science and Manufacturing, 42 (9), 1263-1274.

[7] Zhang, Y., Bai, L. (2015). Rapid structural condition assessment using radio frequency identification (RFID) based wireless strain sensor. Automation in Construction, 54, 1-11.

[8] Sunny, A.I., Tian, G.Y., Zhang, J., Pal, M. (2016). Low frequency (LF) RFID sensors and selective transient feature extraction for corrosion characterisation. Sensors and Actuators A: Physical, 241, 34-43.

[9] Leon-Salas, W.D., Halmen, C. (2016). A RFID sensor for corrosion monitoring in concrete. IEEE Sensors Journal, 16 (1), 32-42.

[10] Bhattacharyya, R., Kalansuriya, P., Sarma, S., Karmakar, N. (2012). Towards chipless RFID-based sensing for pervasive surface crack detection. In 2012 IEEE International Conference on RFID-Technologies and Applications (RFID-TA). IEEE, 46-51.

[11] Caizzone, S., Digiampaolo, E. (2015). Wireless passive RFID crack width sensor for structural health monitoring. IEEE Sensors Journal, 15 (12), 67676774.

[12] Li, Z., Meng, Z. (2016). A review of the radio frequency non-destructive testing for carbon-fibre composites. Measurement Science Review, 16 (2), 68-
76.

[13] Nguyen, D., Phan, G., Pham, T., Le, N. (2013). A battery free RFID sensor for quality detection of food products. In Progress in Electromagnetics Research Symposium Proceedings. PIERS, 583-587.

[14] Potyrailo, R.A., Nagraj, N., Tang, Z., Mondello, F.J., Surman, C., Morris, W. (2012). Battery-free radio frequency identification (RFID) sensors for food quality and safety. Journal of Agricultural and Food Chemistry, 60 (35), 8535-8543.

[15] Donno, D.D., Catarinucci, L., Tarricone, L. (2014). RAMSES: RFID augmented module for smart environmental sensing. IEEE Transactions on Instrumentation and Measurement, 63 (7), 1701-1708.

[16] Tarricone, L. (2013). A long-range computational RFID tag for temperature and acceleration sensing applications. Progress in Electromagnetics Research $C, 45,223-235$.

[17] Catarinucci, L., Colella, R., Tarricone, L. (2009). A cost-effective UHF RFID tag for transmission of generic sensor data in wireless sensor networks. IEEE Transactions on Microwave Theory and Techniques, 57 (5), 1291-1296.

[18] López-Soriano, S., Parrón, J. (2015). Wearable RFID tag antenna for healthcare applications. In 2015 IEEEAPS Topical Conference on Antennas and Propagation in Wireless Communications (APWC). IEEE, 287-290.

[19] Choi, B.-S., Lee, J.-W., Lee, J.-J., Park, K.T. (2011). A hierarchical algorithm for indoor mobile robot localization using RFID sensor fusion. IEEE Transactions on Industrial Electronics, 58 (6), 22262235.

[20] Nikitin, P.V., Rao, K.V.S. (2006). Theory and measurement of backscattering from RFID tags. IEEE Antennas and Propagation Magazine, 48 (6), 212-218.

[21] EPCglobal Inc. (2013). EPC radio-frequency identity protocols Generation-2 UHF RFID. Version 2.0.0 Ratified.

[22] Powercast Corporation. (2010). P2110 - $915 \mathrm{MHz} R \mathrm{RF}$ power harvester receiver. REV A - 2014/11.

[23] Donno, D.D., Catarinucci, L., Tarricone, L. (2014). A battery-assisted sensor-enhanced RFID tag enabling heterogeneous wireless sensor networks. IEEE Sensors Journal, 14 (4), 1048-1055.

[24] Kruesi, C. (2009). Design and development of a novel 3-D cubic antenna for wireless sensor networks (WSNs) and RFID applications. IEEE Antennas and Propagation Magazine, 57 (10), 3293-3299.

[25] Huber, T., et al. (2014). Ultra-low-cost RFID based on soft magnetic ribbons. IEEE Transactions on Magnetics, 50 (10), 1-5.

[26] Huang, X., et al. (2015). Binder-free highly conductive graphene laminate for low cost printed radio frequency applications. Applied Physics Letters, 106 (20), 203105.

[27] Shao, S., Kiourti, A., Burkholder, R.J., Volakis, J.L. (2015). Broadband textile-based passive UHF RFID tag antenna for elastic material. IEEE Antennas and 
Wireless Propagation Letters, 14, 1385-1388.

[28] Yang, L., Martin, L.J., Staiculescu, D., Wong, C.P., Tentzeris, M.M. (2008). Conformal magnetic composite RFID for wearable RF and bio-monitoring applications. IEEE Transactions on Microwave Theory and Techniques, 56 (12), 3223-3230.

[29] Tedjini, S., Karmakar, N., Perret, E., Vena, A., Koswatta, R., E-Azim, R. (2013). Hold the chips: Chipless technology, an alternative technique for RFID. IEEE Microwave Magazine, 14 (5), 56-65.

[30] Kalansuriya, P., Karmakar, N.C., Viterbo, E. (2012). On the detection of frequency-spectra-based chipless RFID using UWB impulsed interrogation. IEEE Transactions on Microwave Theory and Techniques, 60 (12), 4187-4197.

[31] Balbin, I., Karmakar, N.C. (2009). Phase-encoded chipless RFID transponder for large scale low cost applications. IEEE Microwave and Wireless Components Letters, 19 (8), 509-511.

[32] Singh, T., Tedjini, S., Perret, E., Vena, A. (2011). A frequency signature based method for the RF identification of letters. In 2011 IEEE International Conference on RFID. IEEE, 1-5.

[33] You, K., Kim, H., Kim, M., Yang, Y. (2011). 900 $\mathrm{MHz}$ CMOS RF-to-DC converter using a crosscoupled charge pump for energy harvesting. In 2011 IEEE International Symposium on Radio-Frequency Integration Technology (RFIT). IEEE, 149-152.

[34] Ramos, A., Girbau, D., Lázaro, A., Collado, A., Georgiadis, A. (2015). Solar-powered wireless temperature sensor based on UWB RFID with selfcalibration. IEEE Sensors Journal, 15 (7), 3764-3772.

[35] Xiao, Z., et al. (2015). An implantable RFID sensor tag toward continuous glucose monitoring. IEEE Journal of Biomedical and Health Informatics, 19 (3), 910-919.

[36] Salmeron, J.F., et al. (2014). Design and development of sensing RFID tags on flexible foil compatible with EPC gen 2. IEEE Sensors Journal, 14 (12), 43614371.

[37] Law, M.K., Bermak, A., Luong, H.C. (2010). A sub- $\mu$ W embedded CMOS temperature sensor for RFID food monitoring application. IEEE Journal of SolidState Circuits, 45 (6), 1246-1255.

[38] Kellomäki, T. (2012). On-body performance of a wearable single-layer RFID tag. IEEE Antennas and Wireless Propagation Letters, 11, 73-76.

[39] Occhiuzzi, C., Cippitelli, S., Marrocco, G. (2010). Modeling, design and experimentation of wearable RFID sensor tag. IEEE Transactions on Antennas and Propagation, 58 (8), 2490-2498.

[40] Kalansuriya, P., Bhattacharyya, R., Sarma, S. (2013). RFID tag antenna-based sensing for pervasive surface crack detection. IEEE Sensors Journal, 13 (5), 15641570 .

[41] Murthy, S.G.N. (2015). Batteryless Wireless RFID based embedded sensors for long term monitoring of reinforced concrete structures. In 2015 International
Symposium Non-Destructive Testing in Civil Engineering, 1-8.

[42] Kim, J., Wang, Z., Kim, W.S. (2014). Stretchable RFID for wireless strain sensing with silver nano ink. IEEE Sensors Journal, 14 (12), 4395-4401.

[43] Hasani, M., Vena, A., Sydänheimo, L., Ukkonen, L., Tentzeris, M.M. (2013). Implementation of a dual interrogation mode embroidered RFID-enabled strain sensor. IEEE Antennas and Wireless Propagation Letters, 12, 1272-1275.

[44] Manzari, S., Catini, A., Pomarico, G., Di Natale, C., Marrocco, G. (2014). Development of an UHF RFID chemical sensor array for battery-less ambient sensing. IEEE Sensors Journal, 14 (10), 3616-3623.

[45] Occhiuzzi, C., Rida, A., Marrocco, G., Tentzeris, M.M. (2011). CNT-based RFID passive gas sensor. In 2011 IEEE MTT-S International Microwave Symposium Digest. IEEE, 1-4.

[46] Yang, L., Zhang, R., Staiculescu, D. (2009). A novel conformal RFID-Enabled module utilizing inkjetprinted antennas and carbon nanotubes for gasdetection applications. IEEE Antennas and Wireless Propagation Letters, 8, 653-656.

[47] Jia, Y., Heiß, M., Fu, Q., Gay, N.A. (2009). A prototype RFID humidity sensor for built environment monitoring. In International Workshop on Education Technology and Training and International Workshop on Geoscience and Remote Sensing (ETT and GRS 2008). IEEE, 496-499.

[48] Qi, Z., Zhuang, Y., Li, X., Liu, W., Du, Y., Wang, B. (2014). Full passive UHF RFID tag with an ultra-low power, small area, high resolution temperature sensor suitable for environment monitoring. Microelectronics Journal, 45 (1), 126-131.

[49] Tan, Z., et al. (2013). A 1.2-V 8.3-nJ CMOS humidity sensor for RFID applications. IEEE Journal of SolidState Circuits, 48 (10), 2469-2477.

[50] Wu, X., Deng, F., Hao, Y., Fu, Z., Zhang, L. (2015). Design of a humidity sensor tag for passive wireless applications. Sensors, 15 (10), 25564-25576.

[51] Feng, Y., Xie, L., Chen, Q., Zheng, L.-R. (2015). Low-cost printed chipless RFID humidity sensor tag for intelligent packaging. IEEE Sensors Journal, 15 (6), 3201-3208.

[52] Fernandez-Salmeron, J., et al. (2015). HF RFID tag as humidity sensor: Two different approaches. IEEE Sensors Journal, 15 (10), 5726-5733.

[53] Luvisi, A., Panattoni, A., Materazzi, A. (2016). RFID temperature sensors for monitoring soil solarization with biodegradable films. Computers and Electronics in Agriculture, 123, 135-141.

[54] Yin, J., et al. (2010). A system-on-chip EPC Gen-2 passive UHF RFID tag with embedded temperature sensor. IEEE Journal of Solid-State Circuits, 45 (11), 2404-2420.

[55] Fiddes, L.K., Chang, J., Yan, N. (2014). Electrochemical detection of biogenic amines during food spoilage using an integrated sensing RFID tag. 
Sensors and Actuators B: Chemical, 202, 1298-1304.

[56] Eom, K.H., Kim, M.C., Lee, S.J., won Lee, C. (2012). The vegetable freshness monitoring system using RFID with oxygen and carbon dioxide sensor. International Journal of Distributed Sensor Networks, 8 (6), 472986.

[57] Le, G.T., Tran, T.V., Lee, H.-S., Chung, W.-Y. (2016). Long-range batteryless RF sensor for monitoring the freshness of packaged vegetables. Sensors and Actuators A: Physical, 237, 20-28.

[58] Hyun, K.H., Lee, C.W., Kim, J.W., Eom, K.H. (2014). Food monitoring system using $15.36 \mathrm{MHz}$ and $900 \mathrm{MHz}$ smart RFID tag. Advanced Science and Technology Letters, 49, 136-143.

[59] Badia-Melis, R., Ruiz-Garcia, L., Garcia-Hierro, J., Villalba, J.I.R. (2015). Refrigerated fruit storage monitoring combining two different wireless sensing technologies: RFID and WSN. Sensors, 15 (3), 4781 4795.

[60] Saravanan, M.S., Singh, J.K., Thirumoorthy, N. (2014). RFID sensors for food safety centre by identifying the physical factors that affecting the food. In 2014 International Conference on Information Communication and Embedded Systems (ICICES). IEEE, 1-6.

[61] Wang, J., Ni, D., Li, K. (2014). RFID-based vehicle positioning and its applications in connected vehicles. Sensors, 14 (3), 4225-4238.

[62] Shirehjini, A.A.N., Yassine, A., Shirmohammadi, S. (2012). Equipment location in hospitals using RFIDbased positioning system. IEEE Transactions on Information Technology in Biomedicine, 16 (6), 10581069.

[63] Miah, M.S., Gueaieb, W. (2014). Mobile robot trajectory tracking using noisy RSS measurements: An RFID approach. ISA Transactions, 53 (2), 433-443.

[64] Seok, J.-H., Lee, J.-Y., Oh, Ch., Lee, J.-J., Lee, H.J. (2010). RFID sensor deployment using differential evolution for indoor mobile robot localization. In 2010 IEEE/RSJ International Conference on Intelligent Robots and Systems (IROS). IEEE, 3719-3724.

[65] Liu, G., Mao, L., Chen, L., Xie, S. (2014). Locatablebody temperature monitoring based on semi-active UHF RFID tags. Sensors, 14 (4), 5952-5966.

[66] Song, X., Li, X., Tang, W., Zhang, W., Li, B. (2014). A hybrid positioning strategy for vehicles in a tunnel based on RFID and in-vehicle sensors. Sensors, 14 (12), 23095-23118.

[67] Huang, C.-H., Lee, L.-H., Ho, C.C., Wu, L.-L., Lai, Z.-H. (2015). Real-time RFID indoor positioning system based on Kalman-filter drift removal and Heron-bilateration location estimation. IEEE Transactions on Instrumentation and Measurement, 64 (3), 728-739.
[68] Dian, Z., Kezhong, L., Rui, M. (2015). A precise RFID indoor localization system with sensor network assistance. China Communications, 12 (4), 13-22.

[69] Cangialosi, A., Monaly, J.E., Yang, S.C. (2007). Leveraging RFID in hospitals: Patient life cycle and mobility perspectives. IEEE Communications Magazine, 45 (9), 18-23.

[70] Vaz, A., et al. (2010). Full passive UHF tag with a temperature sensor suitable for human body temperature monitoring. IEEE Transactions on Circuits and Systems II: Express Briefs, 57 (2), 95-99.

[71] Rakibet, O.O., Rumens, C.V., Batchelor, J.C., Holder, S.J. (2014). Epidermal passive RFID strain sensor for assisted technologies. IEEE Antennas and Wireless Propagation Letters, 13, 814-817.

[72] Rose, D.P., et al. (2015). Adhesive RFID sensor patch for monitoring of sweat electrolytes. IEEE Transactions on Biomedical Engineering, 62 (6), 1457-1465.

[73] Wickramasinghe, A., Ranasinghe, D.C. (2015). Ambulatory monitoring using passive computational RFID sensors. IEEE Sensors Journal, 15 (10), 58595869.

[74] Barman, J., et al. (2012). Sensor-enabled RFID system for monitoring arm activity: Reliability and validity. IEEE Transactions on Neural Systems and Rehabilitation Engineering, 20 (6), 771-777.

[75] Wang, L., et al. (2016). Toward a wearable RFID system for real-time activity recognition using radio patterns. IEEE Transactions on Mobile Computing, 6 (1), 1-13.

[76] Smith, B.J.R., et al. (2005). RFID-based techniques for human-activity detection. Communications of the ACM, 48 (9), 39-44.

[77] Potyrailo, R.A., Mouquin, H., Morris, W.G. (2008). Position-independent chemical quantitation with passive $13.56-\mathrm{MHz}$ radio frequency identification (RFID) sensors. Talanta, 75 (3), 624-628.

[78] Ramos, A., Girbau, D., Lazaro, A., Villarino, R. (2015). Wireless concrete mixture composition sensor based on time-coded UWB RFID. IEEE Microwave and Wireless Components Letters, 25 (10), 681-683.

[79] Lei, Z., Zhi, W. (2006). Integration of RFID into wireless sensor networks: Architectures, opportunities and challenging problems. In Fifth International Conference on Grid and Cooperative Computing Workshops (GCCW'06). IEEE, 463-469.

[80] Decarli, N., Guidi, F., Dardari, D., (2016). Passive UWB RFID for tag cocalization: Architectures and design. IEEE Sensors Journal, 16 (5), 1385-1397.

Received June 23, 2016. Accepted November 28, 2016. 